\title{
Gamma-ray burst observation \& gravitational wave event follow-up with CALET on the International Space Station
}

\section{Yuta Kawakubo ${ }^{a, *}$ on behalf of the CALET Collaboration}

(a complete list of authors can be found at the end of the proceedings)

\author{
${ }^{a}$ Department Physics \& Astronomy, Louisiana State University, \\ 202 Nicholson Hall, Baton Rouge, LA 70803, USA \\ E-mail: kawakubo1@lsu.edu
}

\begin{abstract}
The CALorimetric Electron Telescope (CALET) has been observing high-energy cosmic rays and gamma-rays on the International Space Station since October 2015. The CALET gamma-ray burst monitor (CGBM), mounted on CALET to observe prompt emissions of gamma-ray bursts (GRBs) in the hard X-ray and soft gamma-ray band, has been monitoring all-sky with $~ 60 \%$ duty cycle without any problems since October 2015. As of end May 2021, CGBM has detected 254 GRBs, including 31 short GRBs, thanks to the onboard trigger system. The Calorimeter (CAL), the primary instrument of CALET, has also collected gamma-ray data in the energy range from $1 \mathrm{GeV}$ to $10 \mathrm{TeV}$ while maintaining both instruments in good condition. We continue searching for highenergy gamma-rays from GRBs detected by CGBM, and have found two possible gamma-rays from GRBs. As described above, CALET can detect prompt emissions and high energy gammaray emission of GRBs. Therefore, we also have actively participated in the follow-up campaign for electromagnetic counterparts of the gravitational wave events observed by LIGO/Virgo since the operation start of the CALET. Although we have found no candidates of electromagnetic counterparts of the gravitational wave events, we have derived upper limits of the high-energy gamma-ray flux for 26 events in the LIGO/Virgo third observation run.
\end{abstract}

$37^{\text {th }}$ International Cosmic Ray Conference (ICRC 2021)

July 12th - 23rd, 2021

Online - Berlin, Germany

\footnotetext{
${ }^{*}$ Presenter
} 


\section{Introduction}

Gamma-ray bursts (GRB) have been observed by many telescopes in space and on the ground in various wavelengths since the discovery by the Vela satellite [1][2]. In recent years, GRBs, especially short GRBs, have taken on increased importance because a short GRB is a plausible candidate for gravitational wave events' electromagnetic counterparts. Fermi-GBM and INTEGRAL SPI-ACS detected GRB 170817A in association with binary neutron star merger GW 170817 [4][5]. Since GRB 170817A is the only GRB observed and associated with a gravitational wave event, further detection of GRBs associated with gravitational wave events is essential to understand the association between binary neutron star mergers and short GRBs, and the short GRB itself.

Since the coverage by a single GRB instrument is limited, observing GRBs with multiple instruments increases the validity of the observation and the chance of detecting GRBs associated with gravitational wave events. The CALorimetric Electron Telescope (CALET) is a payload on the International Space Station [7]. CALET consists of the Calorimeter (CAL) and CALET Gammaray burst monitor (CGBM). CALET has been monitoring all-sky with X-ray and gamma-rays. In particular, CGBM has been detecting GRBs with a rate of $\sim 45$ GRBs / year. In this paper, we will present an overview of GRB observations with CALET in five years. Also, we will present a summary of follow-up observations for electromagnetic counterparts of gravitational wave events in the LIGO/Virgo third observation run. Detailed gamma-ray observations of CAL will be presented in different papers in ICRC 2021 [8][9].

\section{GRB observation with CALET}

In GRB observations with CALET, CGBM is primarily responsible for observing prompt emission of GRBs. CGBM consists of two Hard X-ray Monitors (HXMs) and a Soft Gamma-ray Monitor (SGM) [10][11]. Both HXM and SGM are scintillation detectors that have $\mathrm{LaBr}_{3}(\mathrm{Ce})$ and BGO for each. CGBM covers the energy range from $7 \mathrm{keV}$ to $20 \mathrm{MeV}$ thanks to $\mathrm{HXM}(7 \mathrm{keV}$ $1 \mathrm{MeV}$ ) and SGM (40 keV - $20 \mathrm{MeV})$. CGBM collects two types of monitor data continuously: Time History $(\mathrm{TH})$ data, which have eight energy channels and are collected every $1 / 8 \mathrm{~s}$, and Pulse Height (PH) data, which have 512 energy channels, are collected every 4 s. CGBM has an onboard trigger system to detect GRBs and other X-ray/gamma-ray transients. The onboard trigger system calculates the signal-to-noise ratio (SNR) according to (1)

$$
\mathrm{SNR}=\frac{N_{\mathrm{tot}}-\frac{N_{\mathrm{BG}}}{\Delta t_{\mathrm{BG}}} \Delta t}{\sqrt{\frac{N_{\mathrm{BG}}}{\Delta t_{\mathrm{BG}}} \Delta t}}
$$

where $\Delta t$ is the integration time of the foreground $(1 / 4 \mathrm{~s}, 1 / 2 \mathrm{~s}, 1 \mathrm{~s}$, and $4 \mathrm{~s}) ; \Delta t_{\mathrm{BG}}$ is integration time of the background $(8,16,32,64 \mathrm{~s}) . N_{\text {tot }}$ is integrated counts over $\Delta t$ in the selected energy range, and $N_{\mathrm{BG}}$ is integrated counts over $\Delta t_{\mathrm{BG}}$ in the selected energy range. In the flight operation, trigger thresholds $(\sigma)$ are selected from $4,5.5,7,8.5,10,11$, and 13 for each $\Delta t . \Delta t_{\mathrm{BG}}$ is selected from $8 \mathrm{~s}$, $16 \mathrm{~s}, 32 \mathrm{~s}, 64 \mathrm{~s}$ and used for all $\Delta t$ conditions. $\Delta t_{\mathrm{BG}}$ is taken from a time interval before $\Delta t$. Since CGBM has three sensors, and there are four $\Delta t$ conditions, SNRs are calculated every $1 / 4 \mathrm{~s}$ in the twelve trigger conditions in parallel. If any SNRs exceed trigger thresholds, CGBM captures event 
data that have higher time and energy resolution than those of monitor data. The onboard CGBM buffer is able to store four events. If four triggers occur before downlink, the onboard trigger is disabled until the event data is transferred and deleted from the buffer. Also, the ground analysis server analyzes real-time TH data and sends an alert as a GCN notice when a CGBM onboard trigger occurs. The trigger settings as of May 31, 2021, are shown in Table 1.

Figure 1 shows averaged count rate maps of each CGBM detector. Background count rates due to charged particles highly depend on geomagnetic position. CGBM count rates increase at the high latitude and around the South Atlantic Anomaly (SAA). CGBM high voltages are turned off at high latitude and around the SAA to avoid high count rates and false triggers due to charged particles. As a result, the duty cycle of CGBM is $\sim 60 \%$. Since background count rates vary depending on time, CGBM is triggered by high count rates due to charged particles sometimes, even if the CGBM high voltages are turned off at the high count rate region. Since HXM has a sensitivity to X-ray below $10 \mathrm{keV}$, bright X-ray sources in the HXM field of view cause increased HXM count rates. Figure 2 shows background spectra observed by each CGBM detector on October 5 in 2015 and April 5 in 2021. CGBM has been collecting X-ray and gamma-rays data without any problem for more than five years since the operation start. All CGBM detectors can see the $511 \mathrm{keV}$ line due to annihilation. Two internal background lines can be seen around $35 \mathrm{keV}$ and $1470 \mathrm{keV}$ in the HXM background spectra [12]. The $2.2 \mathrm{MeV}$ line originating from activation can be seen in the SGM background spectra [13]. These background lines can be used for energy calibration.

At the end of May 2021, CGBM has detected 254 GRBs thanks to the onboard trigger system. The total observation interval is 2066 days, and the GRB detection rate is 44.9 GRBs / year. Figure 3 shows the $T_{90}$ distribution of GRBs detected by CGBM. $T_{90}$ was measured by SGM in the energy range $40 \sim 1000 \mathrm{keV}$ using 'battblocks', which is software for Swift-BAT data to measure the duration using the Bayesian block method [14]. Although TH data were used for $T_{90}$ calculation, event data were used if calculated $T_{90}$ was less than $2 \mathrm{~s}$. Since 5 out of 254 GRBs were low significance or not seen in SGM data, 249 GRBs were included in Figure 3. The $T_{90}$ distribution by CGBM is bimodal in logarithmic scale like those by other instruments, and well fitted with two logarithmic normal distributions. The means of the two logarithmic normal distributions were $0.51 \mathrm{~s}$ and $16.98 \mathrm{~s}$. The intersection of the two logarithmic normal distributions was $1.44 \mathrm{~s}$. If we classify GRBs by $1.44 \mathrm{~s}$, the number of long bursts and short bursts were 218 and 31, respectively. Also, the five GRBs for which SGM did not measure $T_{90}$ were long GRBs judging from HXM data. Therefore, $12.4 \%$ of GRBs seen by CGBM were short GRBs. Figure 4 shows the GRB position in SGM coordinates with regions obstructed by fixed structures. Since CGBM has no capability of GRB localization, we used GRB positions that were reported to GCN by other GRB instruments [15]. 182 out of 254 GRBs were localized by other GRB instruments and included in Figure 4.

Table 1: Settings for CGBM onboard trigger

\begin{tabular}{lcc}
\hline \hline & HXM & SGM \\
\hline Trigger threshold $\sigma$ & 8.5 & 7.0 \\
$\Delta t_{\mathrm{BG}}$ & $16 \mathrm{~s}$ & $16 \mathrm{~s}$ \\
Energy range & $25 \sim 100 \mathrm{keV}$ & $50 \sim 300 \mathrm{keV}$ \\
\hline
\end{tabular}



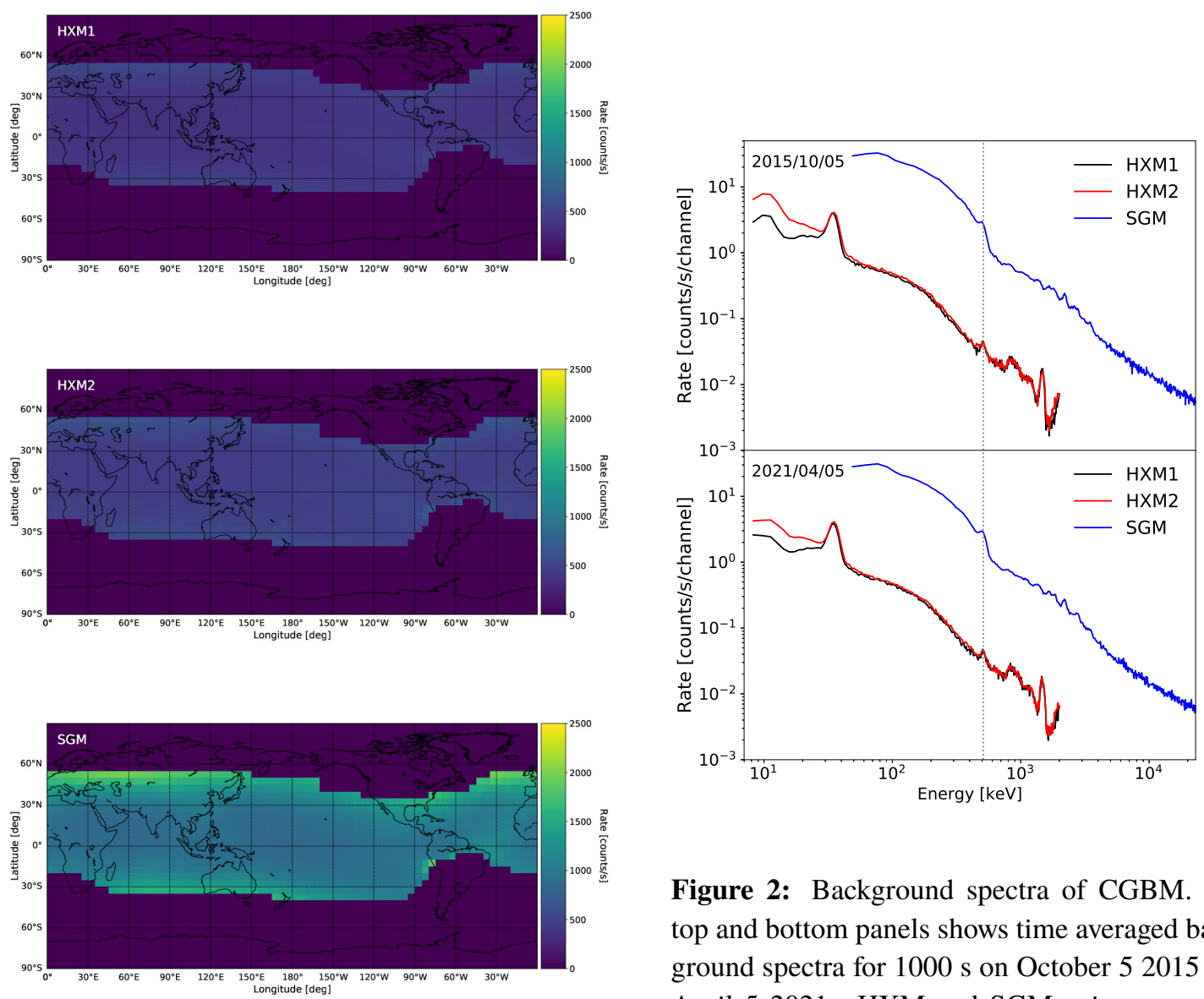

Figure 2: Background spectra of CGBM. The top and bottom panels shows time averaged background spectra for $1000 \mathrm{~s}$ on October 52015 and April 5 2021. HXM and SGM gains were corrected by the position of $1.4 \mathrm{MeV}$ and $2.2 \mathrm{MeV}$

Figure 1: Background count rate maps measured by CGBM. The top, middle, and bottom panels are averaged count rate maps for each geographic position lines for each. Gray dotted lines show the position of $511 \mathrm{keV}$. for HXM1, HXM2 and SGM, respectively. The count rates were calculated using $\mathrm{PH}$ data for September 2020 and averaged in each 5 deg. pixel.

Since CALET is not a satellite but a payload on the ISS, ISS structures obstruct the CAL and CGBM fields of view. In addition to fixed structures, there are both regularly transient structures (e.g. solar panels and radiators) and irregularly transient structures (e.g. robotic arms). Most GRBs detected by CGBM arrived from the direction not obstructed by the fixed structures. However, some GRBs arrived from the obstructed region. Although localization errors were ignored, there is $5-15 \mathrm{deg}$ uncertainty for each point. Also, there is a possibility that ISS structures might have gaps. Full effects of the ISS structures on CGBM data are unclear, and we continue to investigate this issues.

In the gamma-ray analysis with CAL, data collected in the high energy trigger (HE) mode and low energy gamma-ray (LEG) mode are used for the analysis above $10 \mathrm{GeV}$ and $1 \mathrm{GeV}$, respectively [16]. The HE mode is the primary trigger mode of CAL. The HE mode is available anytime except when CAL is collecting pedestal data. LEG mode is enabled only at low latitude, or for a short 


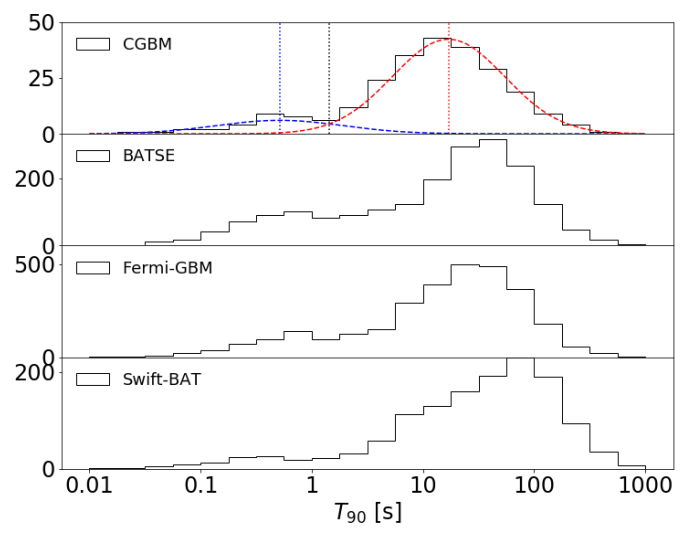

Figure 3: $T_{90}$ distribution of CGBM GRBs. Energy ranges of CGBM, BATSE, Fermi-GBM, and Swift-BAT are 40 $1000 \mathrm{keV}, 25 \sim 2000 \mathrm{keV}, 50 \sim 300 \mathrm{keV}$, and $15 \sim 350 \mathrm{keV}$, respectively [17-21]. Blue and red dashed lines are two optimized logarithmic normal distributions. Blue and red dotted lines show mean of the two distributions. Gray dotted line shows the intersection of the two distributions.

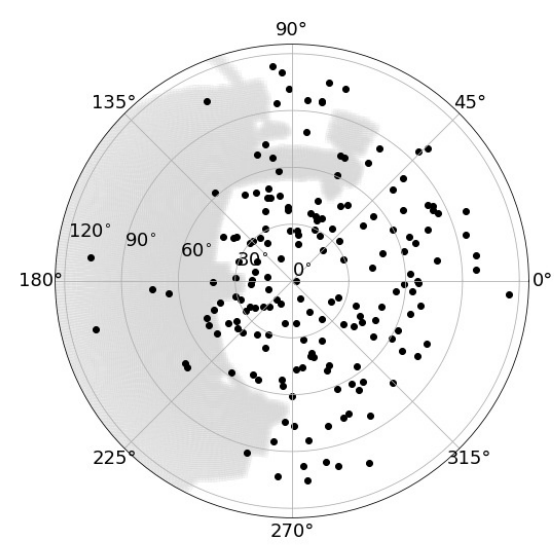

Figure 4: Incident angle distribution of GRBs in the SGM field of view. Black points are GRB positions in the SGM coordinate. A gray shaded region is the ISS fixed structure viewed from CALET.

period when a CGBM onboard trigger occurs [22]. We have continued to search for high-energy gamma-rays using CAL data [23]. We searched for high-energy gamma-rays from GRBs detected by CGBM using CAL data up to September 30 in 2020. We searched for gamma-ray events in $1 \mathrm{GeV} \sim 10 \mathrm{GeV}$ for $99 \mathrm{GRBs}$ which were well localized by Swift-BAT, XRT, UVOT, Fermi-LAT, MAXI-GSC, and IPN, using LEG data from $T_{0}-60 \mathrm{~s}$ to $T_{0}+7200 \mathrm{~s}$ within $2 \mathrm{deg}$ from the reported GRB central position, where $T_{0}$ is the trigger time of CGBM. The gamma-ray identification was performed according to the method described in [16]. The directions obstructed by fixed structures and moving structures except transient obstruction (e.g., robotic arms), were masked to exclude secondary gamma-rays from the structures. In the case of gamma-ray candidates from GRBs were found, we checked the effects of transient obstruction for each candidate by making scatter plots of arrival directions of gamma-ray candidates on the detector coordinate. As a result, the GRB positions were outside of the CAL field of view, or there was no available LEG data for 37 GRBs. There was no gamma-ray event near the GRB positions for 59 GRBs, even if the GRB positions were in the CAL field of view. Gamma-ray events were found near the position of GRB 180526A, GRB 200101A, and GRB 200613A. In the case of GRB 200613A, we found the robotic arm obstructed the CAL field of view on June 13, 2020, and we concluded the gamma-ray event was a secondary gamma-ray event from the obstructions. CGBM detected GRB 180526A at $T_{0}=$ 2018/05/26 11:03:36.20 UT. A $3.4 \mathrm{GeV}$ gamma-ray event was found at $1.3 \mathrm{deg}$. away from the reported position (R.A., Dec.) $=(108.48$ deg., 3.64 deg. $)$ by Fermi-LAT at $T_{0}+244 \mathrm{~s}$ [24] . The central position of GRB 180526A was within the $99 \%$ PSF region of the candidate. CGBM also detected GRB 200101A at $T_{0}=2020 / 01 / 01$ 20:39:30.40 UT. A 4.9 GeV gamma-ray event was found at 0.6 deg. away from the reported position (R.A., Dec.) $=(258.995$ deg., -32.304 deg. $)$ by IPN at $T_{0}+105 \mathrm{~s}$ [15] (\#26635). The central position of GRB 200101A was within the $90 \%$ PSF region 
Table 2: Summary of follow-up observation for gravitational wave events in $\mathrm{O} 3$

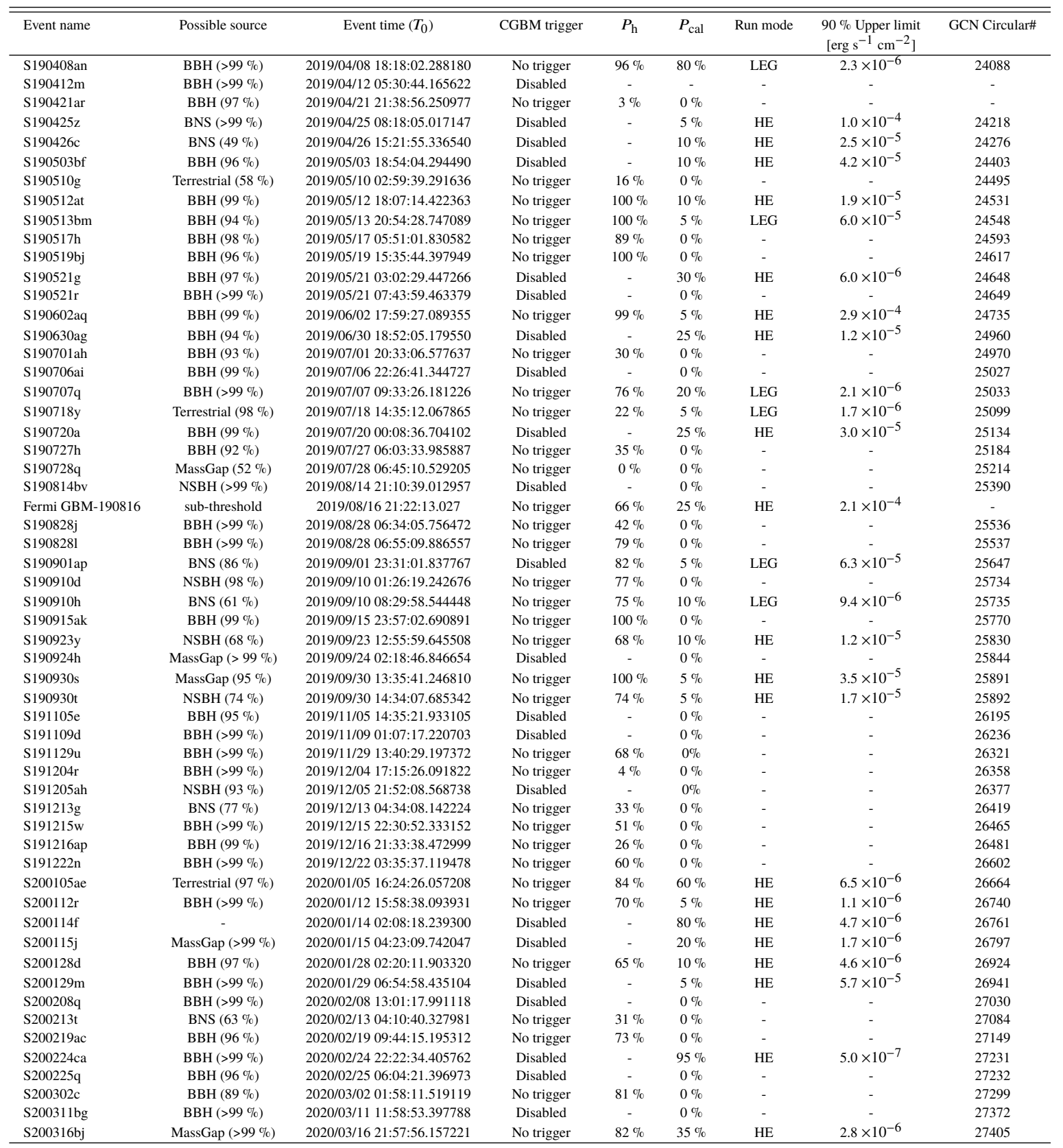

of the candidate. No excess can be seen in CGBM at the arrival time of the candidates for GRB 180526A and GRB 200101A. A detailed analysis is still underway.

\section{Follow-up for gravitational wave events in LIGO/Virgo 03}

CALET participated in the follow-up campaign for the LIGO/Virgo first and second observation runs [25][26][6]. Also, we performed a follow-up observation of electromagnetic counterparts of the gravitational wave using both CAL and CGBM data in the LIGO/Virgo third observation run 
(O3) [27] [11]. Table 2 shows a summary of CALET follow-up observations. There are 56 events reported by the LIGO/Virgo collaboration (LVC) and one sub-threshold event reported by LVC and Fermi-GBM team[28] [15]. 'Event name,' 'Possible source,' and 'Event time $\left(T_{0}\right)$ ' are based on GraceDB and GCN circulars reported by the LVC and Fermi-GBM team [28][15](\#25406). The 'Possible source' column shows just the highest probability source in the GCN circulars.

'CGBM trigger' shows the status of the CGBM onboard trigger at $T_{0}$. 'No trigger' means that CGBM onboard trigger was enabled; however, no trigger occurred in $T_{0}-60 \mathrm{~s} \sim T_{0}+60 \mathrm{~s}$. 'Disabled' means the CGBM onboard trigger was disabled due to CGBM high voltages were off, or the CGBM event data storage was full. There were no onboard triggers associated with any gravitational wave events. ' $P$ ' ' shows the summed LIGO/Virgo localization probability above the horizon. If the CGBM high voltages were off, the column was filled by '-.' We also searched for electromagnetic signals in TH data for 36 events that occurred when the CGBM high voltage was on. We calculated the SNRs using (1) with extended conditions and searched for significant signals according to the method described in [11] using TH data for $T_{0}-60 \mathrm{~s} \sim T_{0}+60 \mathrm{~s}$, where $T_{0}$ is the trigger time of the gravitational wave event. As a result, there was no significant signal associated with the gravitational wave events in the CGBM data.

' $P_{\text {cal }}$ ' shows the summed LIGO/Virgo localization probability in the CAL field of view for $T_{0}-60 \mathrm{~s} \sim T_{0}+60 \mathrm{~s}$. Although CAL's high voltages are typically always on, CALET was off due to a special activity on ISS when S190412 occurred. 'Run mode' shows the CAL run mode at $T_{0}$. If $P_{\text {cal }}$ is zero, the column was filled by '-.' In the case where $P_{\text {cal }}$ was $5 \%$ or greater, we searched for gamma-ray events from the LIGO/Virgo localization high probability region in $\mathrm{HE}$ (10 GeV $100 \mathrm{GeV})$ or LEG $(1 \mathrm{GeV} \sim 10 \mathrm{GeV})$ data for $T_{0}-60 \mathrm{~s} \sim T_{0}+60 \mathrm{~s}$. Although there was no high energy gamma-ray candidate associated with the gravitational wave events, we estimated $90 \%$ upper limits of gamma-ray flux for each direction according to the method described in [26][27]. The $90 \%$ upper limits were calculated for the energy range $10 \mathrm{GeV} \sim 100 \mathrm{GeV}$ and $1 \mathrm{GeV} \sim 10 \mathrm{GeV}$ in the case of HE and LEG data, respectively. As examples of the analysis, Figure 5 shows the $90 \%$ upper limits maps for S190408an and S200316bj. Since effective areas for small incident angles are larger than that for large incident angles, stricter upper limits are derived near the CALET zenith than near the edge of the CAL field of view. The dented structures around the edge of the CAL field of view were masked due to the fixed ISS structures. '90 \% Upper limit' shows the highest $90 \%$ upper limits of gamma-ray flux when the summed LIGO/Virgo localization probability reached $P_{\text {cal }}$.

\section{Summary}

CALET has been in in-orbit operation since October 2015 without any problems. CGBM has been continuing all-sky monitoring of GRBs with a $\sim 60 \%$ duty cycle and observed 254 GRBs, including 31 short GRBs, by the end of May 2021. As the result of high energy gammaray search from GRBs detected by CGBM using CAL, two gamma-ray candidates were found from GRB 180526A and GRB 200101A. CALET also participated in the follow-up campaign for electromagnetic counterparts of gravitational wave events in O3. Although there was no candidate of the electromagnetic counterparts, we estimated $90 \%$ upper limits of gamma-ray flux for 26 gravitational wave events using CAL data. 


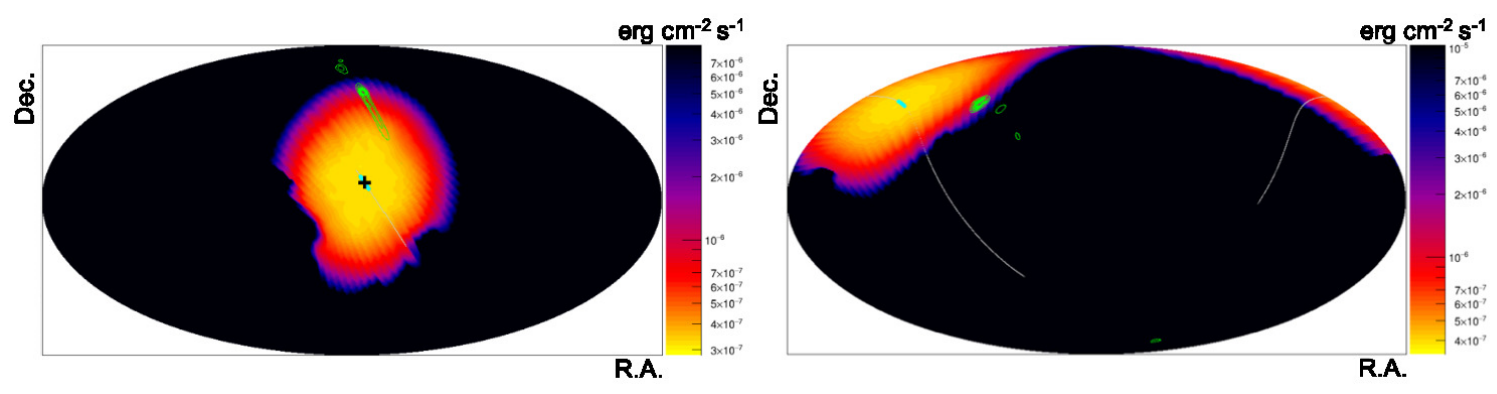

Figure 5: The $90 \%$ upper limits for S190408an (left) and S200316bj (right). The color maps show the 90 $\%$ upper limits of gamma-ray flux. In the case of S190408A, the energy range is $1 \mathrm{GeV} \sim 10 \mathrm{GeV}$. In the case of S200316bj, energy range is $10 \mathrm{GeV} \sim 100 \mathrm{GeV}$. Green contours are the LIGO/Virgo localization high probability region. Black bold cross is the CAL zenith at $T_{0}$. Cyan bold lines are tracks of the CAL zenith for $T_{0}-60 \mathrm{~s} \sim T_{0}+60 \mathrm{~s}$.

\section{References}

[1] R. Y. Klebesadel et al., OBSERVATIONS OF GAMMA-RAY BURSTS OF COSMIC ORIGIN, THE ASTROPHYSICAL JOURNAL, 182, L85, (1973)

[2] P. Kumar, and B. Zhang, The physics of gamma-ray bursts \& relativistic jets, Physics Report, 561, 1, (2015).

[3] C. Kouveliotou et al., IDENTIFICATION OF TWO CLASSES OF GAMMA-RAY BURSTS, THE ASTROPHYSICAL JOURNAL, 413, L101, (1993).

[4] B. P. Abbott et al., GW170817: Observation of Gravitational Waves from a Binary Neutron Star Inspiral, PHYSICAL REVIEW LETTERS, 119, 161101, (2017).

[5] B. P. Abbott et al., Gravitational Waves and Gamma-Rays from a Binary Neutron Star Merger: GW170817 and GRB 170817A, THE ASTROPHYSICAL JOURNAL LETTERS, 848, L13, (2017).

[6] B. P. Abbott et al., Multi-messenger Observations of a Binary Neutron Star Merger, The Astrophysical Journal Letters, 848, L12, (2017).

[7] Y Asaoka for the CALET Collaboration, The CALorimetric Electron Telescope (CALET) on the International Space Station, Proc.36th ICRC (Madison, USA, 2019), POS(ICRC2019), 001, (2019).

[8] N. Cannady on behalf of the CALET Collaboration, Low-energy gamma-ray observations above 1 GeV with CALET on the International Space Station, in this conference.

[9] M. Mori on behalf of the CALET Collaboration., High-energy gamma-ray observations above $10 \mathrm{GeV}$ with CALET on the International Space Station, in this conference.

[10] K. Yamaoka et al., The CALET Gamma-ray Burst Monitor (CGBM)) Proc. 7th Huntsville Gamma-Ray Burst Symposium (Nashville, USA, 2013), paper 41 in eConf Proceedings C1304143, (2013).

[11] Y. Kawakubo et al., Search for electromagnetic counterparts of gravitational wave sources with CALET, Proc. GAMMA-RAY BURSTS IN THE GRAVITATIONAL WAVE ERA, 31, (2020).

[12] F.G.A. Quarati et al., Study of ${ }^{138}$ La radioactive decays using LaBr 3 scintillators, Nuclear Instruments and Methods in Physics Research A, 683, 46, (2012)

[13] P.R. Truscott et al., Activation of Space-Borne Bismuth Germanate $\gamma$-ray Detectors, IEEE TRANSACTIONS ON NUCLEAR SCIENCE, 42, No.4, (1995)

[14] NASA HEASARC battblocks https://heasarc.gsfc.nasa.gov/ftools/caldb/help/battblocks.html

[15] The Gamma-ray Coordinates Network https://gcn.gsfc.nasa.gov

[16] N. Cannady et al., Characteristics and Performance of the CALorimetric Electron Telescope (CALET) Calorimeter for Gamma-Ray Observations, The Astrophysical Journal Supplement Series, 238, 5, (2018)

[17] D. Gruber et al., THE FERMI GBM GAMMA-RAY BURST SPECTRAL CATALOG: FOUR YEARS OF DATA, THE ASTROPHYSICAL JOURNAL SUPPLEMENT SERIES, 211, 12, (2014).

[18] A. von Kienlin et al., THE SECOND FERMI GBM GAMMA-RAY BURST CATALOG: THE FIRST FOUR YEARS, THE ASTROPHYSICAL JOURNAL SUPPLEMENT SERIES, 211, 13, (2014).

[19] P. N. Bhat et al., THE THIRD FERMI GBM GAMMA-RAY BURST CATALOG: THE FIRST SIX YEARS, THE ASTROPHYSICAL JOURNAL SUPPLEMENT SERIES, 223, 28, (2016).

[20] The BATSE Current Gamma-Ray Burst Catalog, the BATSE GRB Team, https://gammaray .msfc.nasa.gov/batse/grb/catalog/current/

[21] The Swift/BAT Gamma-Ray Burst Catalog, https://swift.gsfc.nasa.gov/results/batgrbcat/

[22] Y. Asaoka et al., On-orbit operations and offline data processing of CALET onboard the ISS, Astroparticle Physics, 100, 29, (2018).

[23] N. Cannady for the CALET collaboration, CALET upper limits on GeV-energy gamma-ray burst emission, Proc.36th ICRC (Madison, USA, 2019), POS(ICRC2019), 557, (2019).

[24] Ajello, et al., A Decade of Gamma-Ray Bursts Observed by Fermi-LAT: The Second GRB Catalog, The Astrophysical Journal, 878, 52, (2019),

[25] O. Adriani et al., CALET UPPER LIMITS ON X-RAY AND GAMMA-RAY COUNTERPARTS OF GW151226, The Astrophysical Journal Letters, 829, L20, (2016),

[26] O. Adriani et al., Search for GeV Gamma-Ray Counterparts of Gravitational Wave Events by CALET, The Astrophysical Journal, 863, 160, (2018).

[27] M. Mori \& Y. Asaoka for the CALET Collaboration, High-Energy Gamma-ray Observations Using the CALorimetric Electron Telescope (CALET) on the ISS, Proc.36th ICRC (Madison, USA, 2019), POS(ICRC2019), 586, (2019)

[28] GraceDB, https://gracedb.ligo.org/superevents/public/03/ 


\section{Full Authors List: CALET Collaboration}

O. Adriani ${ }^{1,2}$, Y. Akaike ${ }^{3,4}$, K. Asano ${ }^{5}$, Y. Asaoka ${ }^{5}$, E. Berti ${ }^{1,2}$, G. Bigongiari 6,7 , W. R. Binns ${ }^{8}$, M. Bongi ${ }^{1,2}$, P. Brogi ${ }^{6,7}$, A. Bruno ${ }^{9,10}$, J. H. Buckley ${ }^{8}$, N. Cannady ${ }^{11,12,13}$, G. Castellini ${ }^{14}$, C. Checchia ${ }^{6}$, M. L. Cherry ${ }^{15}$, G. Collazuol ${ }^{16,17}$, K. Ebisawa ${ }^{18}$, A. W. Ficklin ${ }^{15}$, H. Fuke ${ }^{18}$, S. Gonzi ${ }^{12}{ }^{1}$, T. G. Guzik ${ }^{15}$, T. Hams ${ }^{11}$, K. Hibino ${ }^{19}$, M. Ichimura ${ }^{20}$, K. Ioka $^{21}$, W. Ishizaki ${ }^{5}$, M. H. Israel ${ }^{8}$, K. Kasahara ${ }^{22}$, J. Kataoka ${ }^{23}$, R. Kataoka ${ }^{24}$, Y. Katayose ${ }^{25}$, C. Kato $^{26}$, N. Kawanaka ${ }^{27,28}$, Y. Kawakubo ${ }^{15}$, K. Kobayashi ${ }^{3,4}$, K. Kohri ${ }^{29}$, H. S. Krawczynski ${ }^{8}$, J. F. Krizmanic ${ }^{11,12,13}$, P. Maestro6,7, P. S. Marrocchesi 6,7, A. M. Messineo ${ }^{30,7}$, J.W. Mitchel112, S. Miyake ${ }^{32}$, A. A. Moiseev ${ }^{33,12,13 \text {, }}$ M. Mori ${ }^{34}$, N. Mori ${ }^{2}$, H. M. Motz ${ }^{35}$, K. Munakata ${ }^{26}$, S. Nakahira ${ }^{18}$, J. Nishimura ${ }^{18}$, G. A. de Nolfo ${ }^{9}$, S. Okuno ${ }^{19}$, J. F. Ormes $^{36}$, N. Ospina ${ }^{16,17}$, S. Ozawa ${ }^{37}$, L. Pacini ${ }^{1,14,2}$, P. Papini ${ }^{2}$, B. F. Rauch ${ }^{8}$, S. B. Ricciarini ${ }^{14,2}$, K. Sakai ${ }^{11,12,13 \text {, }}$ T. Sakamoto ${ }^{38}$, M. Sasaki ${ }^{33,12,13}$, Y. Shimizu ${ }^{19}$, A. Shiomi ${ }^{39}$, P. Spillantini ${ }^{1}$, F. Stolzi ${ }^{6,7}$, S. Sugita ${ }^{38}$, A. Sulaj ${ }^{6,7}$, M. Takita ${ }^{5}$, T. Tamura ${ }^{19}$, T. Terasawa ${ }^{40}$, S. Torii ${ }^{3}$, Y. Tsunesada ${ }^{41}$, Y. Uchihori ${ }^{42}$, E. Vannuccini ${ }^{2}$, J. P. Wefel ${ }^{15}$, K. Yamaoka ${ }^{43}$, S. Yanagita ${ }^{44}$, A. Yoshida ${ }^{38}$, K. Yoshida $^{22}$, and W. V. Zober ${ }^{8}$

${ }^{1}$ Department of Physics, University of Florence, Via Sansone, 1, 50019 Sesto, Fiorentino, Italy, ${ }^{2}$ INFN Sezione di Florence, Via Sansone, 1, 50019 Sesto, Fiorentino, Italy, ${ }^{3}$ Waseda Research Institute for Science and Engineering, Waseda University, 17 Kikuicho, Shinjuku, Tokyo 162-0044, Japan, ${ }^{4}$ JEM Utilization Center, Human Spaceflight Technology Directorate, Japan Aerospace Exploration Agency, 2-1-1 Sengen, Tsukuba, Ibaraki 305-8505, Japan, ${ }^{5}$ Institute for Cosmic Ray Research, The University of Tokyo, 5-1-5 Kashiwa-no-Ha, Kashiwa, Chiba 277-8582, Japan, ${ }^{6}$ Department of Physical Sciences, Earth and Environment, University of Siena, via Roma 56, 53100 Siena, Italy, ${ }^{7}$ INFN Sezione di Pisa, Polo Fibonacci, Largo B. Pontecorvo, 3, 56127 Pisa, Italy, ${ }^{8}$ Department of Physics and McDonnell Center for the Space Sciences, Washington University, One Brookings Drive, St. Louis, Missouri 63130-4899, USA, ${ }^{9}$ Heliospheric Physics Laboratory, NASA/GSFC, Greenbelt, Maryland 20771, USA, ${ }^{10}$ Department of Physics, Catholic University of America, Washington, DC 20064, USA, ${ }^{11}$ Center for Space Sciences and Technology, University of Maryland, Baltimore County, 1000 Hilltop Circle, Baltimore, Maryland 21250, USA, ${ }^{12}$ Astroparticle Physics Laboratory, NASA/GSFC, Greenbelt, Maryland 20771, USA, ${ }^{13}$ Center for Research and Exploration in Space Sciences and Technology, NASA/GSFC, Greenbelt, Maryland 20771, USA, ${ }^{14}$ Institute of Applied Physics (IFAC), National Research Council (CNR), Via Madonna del Piano, 10, 50019 Sesto, Fiorentino, Italy, ${ }^{15}$ Department of Physics and Astronomy, Louisiana State University, 202 Nicholson Hall, Baton Rouge, Louisiana 70803, USA, ${ }^{16}$ Department of Physics and Astronomy, University of Padova, Via Marzolo, 8, 35131 Padova, Italy, ${ }^{17}$ INFN Sezione di Padova, Via Marzolo, 8, 35131 Padova, Italy, ${ }^{18}$ Institute of Space and Astronautical Science, Japan Aerospace Exploration Agency, 3-1-1 Yoshinodai, Chuo, Sagamihara, Kanagawa 252-5210, Japan, ${ }^{19}$ Kanagawa University, 3-27-1 Rokkakubashi, Kanagawa, Yokohama, Kanagawa 221-8686, Japan, ${ }^{20}$ Faculty of Science and Technology, Graduate School of Science and Technology,, Hirosaki University, 3, Bunkyo, Hirosaki, Aomori 036-8561, Japan, ${ }^{21}$ Yukawa Institute for Theoretical Physics, Kyoto University, Kitashirakawa Oiwakecho, Sakyo, Kyoto 606-8502, Japan, ${ }^{22}$ Department of Electronic Information Systems, Shibaura Institute of Technology, 307 Fukasaku, Minuma, Saitama 337-8570, Japan, ${ }^{23}$ School of Advanced Science and Engineering, Waseda University, 3-4-1 Okubo, Shinjuku, Tokyo 169-8555, Japan, ${ }^{24}$ National Institute of Polar Research, 10-3, Midori-cho, Tachikawa, Tokyo 190-8518, Japan, ${ }^{25}$ Faculty of Engineering, Division of Intelligent Systems Engineering, Yokohama National University, 79-5 Tokiwadai, Hodogaya, Yokohama 240-8501, Japan, ${ }^{26}$ Faculty of Science, Shinshu University, 3-1-1 Asahi, Matsumoto, Nagano 390-8621, Japan, ${ }^{27}$ Hakubi Center, Kyoto University, Yoshida Honmachi, Sakyo-ku, Kyoto 606-8501, Japan, ${ }^{28}$ Department of Astronomy, Graduate School of Science, Kyoto University, Kitashirakawa Oiwake-cho, Sakyo-ku, Kyoto 6068502, Japan, ${ }^{29}$ Institute of Particle and Nuclear Studies, High Energy Accelerator Research Organization, 1-1 Oho, Tsukuba, Ibaraki 305-0801, Japan, ${ }^{30}$ University of Pisa, Polo Fibonacci, Largo B. Pontecorvo, 3, 56127 Pisa, Italy, ${ }^{31}$ Astroparticle Physics Laboratory, NASA/GSFC, Greenbelt, Maryland 20771, USA, ${ }^{32}$ Department of Electrical and Electronic Systems Engineering, National Institute of Technology, Ibaraki College, 866 Nakane, Hitachinaka, Ibaraki 312-8508, Japan ${ }^{33}$ Department of Astronomy, University of Maryland, College Park, Maryland 20742, USA, ${ }^{34}$ Department of Physical Sciences, College of Science and Engineering, Ritsumeikan University, Shiga 525-8577, Japan, ${ }^{35}$ Faculty of Science and Engineering, Global Center for Science and Engineering, Waseda University, 3-4-1 Okubo, Shinjuku, Tokyo 169-8555, Japan, ${ }^{36}$ Department of Physics and Astronomy, University of Denver, Physics Building, Room 211, 2112 East Wesley Avenue, Denver, Colorado 80208-6900, USA, ${ }^{37}$ Quantum ICT Advanced Development Center, National Institute of Information and Communications Technology, 4-2-1 Nukui-Kitamachi, Koganei, Tokyo 184-8795, Japan, ${ }^{38}$ College of Science and Engineering, Department of Physics and Mathematics, Aoyama Gakuin University, 5-10-1 Fuchinobe, Chuo, Sagamihara, Kanagawa 252-5258, Japan, ${ }^{39}$ College of Industrial Technology, Nihon University, 1-2-1 Izumi, Narashino, Chiba 275-8575, Japan ${ }^{40}$ RIKEN, 2-1 Hirosawa, Wako, Saitama 351-0198, Japan, ${ }^{41}$ Division of Mathematics and Physics, Graduate School of Science, Osaka City University, 3-3-138 Sugimoto, Sumiyoshi, Osaka 558-8585, Japan, ${ }^{42}$ National Institutes for Quantum and Radiation Science and Technology, 4-9-1 Anagawa, Inage, Chiba 263-8555, Japan, ${ }^{43}$ Nagoya University, Furo, Chikusa, Nagoya 464-8601, Japan, ${ }^{44}$ College of Science, Ibaraki University, 2-1-1 Bunkyo, Mito, Ibaraki 310-8512, Japan 